\title{
Nonsurgical Management of Hypertrophic Scars: Evidence-Based Therapies, Standard Practices, and Emerging Methods: An Update
}

\author{
B. Atiyeh $^{1} \cdot$ A. Ibrahim ${ }^{1}$
}

Published online: 5 August 2020

(C) Springer Science+Business Media, LLC, part of Springer Nature and International Society of Aesthetic Plastic Surgery 2020

Repair of injured skin to its original state is of concern to both patient and treating physician. Multiple cells from different lineages are involved in healing of cutaneous wounds. The process is characterized by a complex and sensitive balance between activation and inhibition of inflammation and is well orchestrated and coordinated by signaling transduction of a diversity of growth factors, cytokines, and extracellular matrix (ECM) components $[1,2]$. Unfortunately most skin wound healing following trauma or surgery is imperfect and results in scar formation [1]. Hypertrophic scar (HTS) incidence is $4-16 \%$, and approximately $35 \%$ of all surgical skin wounds result after 1 year in HTSs [3]. Though not life threatening, HTSs cause significant functional, cosmetic, and psychological sequelae [1] often overlooked, scars negatively impact to a great extent the individual's quality of life due to anxiety and depression, loss of self-esteem, stigmatization, and disruption of daily activities [4].

Most described treatment modalities have been proven through extensive use. Though some have achieved acceptance as standard practice, few have been supported by prospective studies with adequate control groups [5]. Review of the vast array of available methods at the time of the initial report demonstrated that most were often inconvenient; besides having occasional undesirable severe side effects with various responses from different patients,

\footnotetext{
B. Atiyeh

bechara.atieh@gmail.com

Beirut, Lebanon
}

they generally did not have consistent efficacy $[4,6]$. There was thus a need to review with evidence-based approach available scar treatment modalities and try to evaluate their efficacy.

Various modalities were identified including application of dynamic and static mechanical forces, pressure garments, light-based therapies, interferon, cryosurgery, radiation, application of topical gels and ointments, and administration of various injectables, including 5-fu, corticosteroids, immune modulators, and hyaluronidases; all have demonstrated limited efficacy [5, 6]. In addition, various emerging cell-based approaches were identified to target different stages of wound healing in an attempt to prevent scar development, but none of these have been entirely successful [6].

An effort was made to distinguish between prophylactic measures and actual treatment methods as well as those modalities with clearly proven efficiency and anecdotal reports about therapies of doubtful benefits [5]. This review concluded that the only treatments for which sufficient evidence existed for the management of a wide variety of abnormal scars to make evidence-based recommendations were silicon gel sheeting and intralesional corticosteroids injection [5].

Since then injection of botulinum toxin type A (BTX-A) and autologous microfat grafting as well as microneedling were added to the armamentarium of HTS management with variable but promising efficacy [7-9]. In a recently published experimental study about the effect of botulinum toxin to which we have personally contributed, it was suggested that the observed benefit of Botox on wound 
healing and scar formation could be due to its profound modulating effect on transforming growth factor beta (TGF-beta1) and tumor necrosis factor alpha (TNF-alpha) expression [10].

Pulsed dye laser (PDL) and fractional ablative $\mathrm{CO}_{2}$ laser are also becoming increasingly popular, with a growing body of research supporting their use for the management of HTSs and keloids. However, the level of evidence of their efficacy remains low [11]. Ablative fractional laser therapies have been shown also to improve the uptake of topical agents into all skin layers in animal experimental models. However, laser-assisted drug delivery (LAD) efficacy still needs to be demonstrated in long-term studies on humans [11].

With skyrocketing healthcare expenditures and recently estimated annual spending on scar management to exceed $\$ 20$ billion in the USA alone [12] and a global scar treatment market size expected to reach a value of $\$ 37.9$ billion by 2026 with a compound annual growth rate (CAGR) of 9.9\% [13], the need to abandon modalities with ill-proven efficacy and select the most appropriate and cost-effective therapies is still as acute. Currently there is a great interest in researching new innovative and more efficacious modalities ultimately inducing tissue regeneration that has functionality and visual appearance as close to the original skin as possible [4].

With a better understanding of the molecular and biologic mechanisms involved in keloid and hypertrophic scar formation, it is becoming evident that a disarray of the three distinct but overlapping phases of wound healing underlines almost all fibrotic conditions [6]. New research has highlighted the importance of intervention at all three phases with multiple simultaneous actions as soon as possible after injury to be continued until wound closure and beyond [4]. It was demonstrated also that injury of tissues with limited regenerative capacity leads to either regeneration or fibrosis, depending on the duration of the inflammatory signal. There is a limited time window of several days in which stopping inflammation avoids fibrosis beyond which fibrosis inevitably occurs even if inflammation is stopped [14]. Understanding the molecular pathways and the myofibroblast-macrophage circuit for wound healing as well as the transition from healing to fibrosis and how injury of tissues with regenerative capacity may lead to two different outcomes, either regeneration or fibrosis, is critical in developing possible new therapeutic strategies for fibrosis and wound healing $[6,14]$.

As our insight into the complex process of tissue regeneration and wound healing increases, new therapeutic modalities will certainly emerge. A recently published study suggests that combining intralesional platelet-rich plasma (PRP) with intralesional triamcinolone acetonide
(TA) could yield cosmetically better outcomes in keloid treatment and probably hypertrophic scars as well with lower incidence of TA-induced atrophy and hypopigmentation [15]. As part of more recent dermal fibroblast studies, it has been shown that dexpanthenol may assist wound healing in all three stages of wound healing by modulating inflammation, supporting cell proliferation, and protecting against infection and free radical damage [4]. Current research targeting inflammatory mediators, epithelial to mesenchymal transition, and regulators of myofibroblast differentiation is showing promising results [15]. Of particular interest though is the recently elucidated diverse role of small leucine-rich proteoglycans (SLRPs), a group of extracellular matrix (ECM), in skin wound healing, such as anti-inflammation, pro-angiogenesis, pro-migration, procontraction, and orchestration of transforming growth factor (TGF-b) signal transduction. This may pave the way for the development of a new generation of pharmaceuticals with beneficial effects on wound healing and final scar formation [1].

Despite increasing clinical concern and tremendous progress in our understanding of wound healing biology and kinetics and despite the many proposed intervention pathways, effective and optimal treatment method for scars and keloids to date is still illusive [11]. With the current state of knowledge, the recommendation remains that treatment of pathologic scars must be individualized and that the combination approach of surgery and adjuvant therapy, such as steroid injections and silicone materials, remains the best option, considering as well promising results of readily available, simple, and cost-effective laser therapy, microneedling and microfat grafting.

\section{References}

1. Pang X, Dong N, Zheng Z (2020) Small leucine-rich proteoglycans in skin wound healing. Front Pharmacol 10:1649

2. Eming SA, Krieg T, Davidson JM (2007) Inflammation in wound repair: molecular and cellular mechanisms. J Invest Dermatol 127:514

3. Zhou Y, Zhao Y, Du H, Suo Y, Chen H et al (2020) Downregulation of CFTR is involved in the formation of hypertrophic scars. Biomed Res Int 2:9526289

4. Baron JM, Glatz M, Proksch E (2020) Optimal support of wound healing: New insights. Dermatology 17:1

5. Atiyeh B (2007) Nonsurgical management of hypertrophic scars: evidence-based therapies, standard practices, and emerging methods. Aesth Plast Surg 31:468

6. El Ayadi A, Jay JW, Prasai A (2020) Current approaches targeting the wound healing phases to attenuate fibrosis and scarring. Int J Mol Sci 7:21

7. Chen S, Yang Y, Zhang D, Liu Y, Li Y (2020) Local injection of botulinum toxin type A to prevent postoperative scar. J Craniofac Surg. https://doi.org/10.1097/scs.0000000000006144. [Epub ahead of print] 
8. Vijaya Lakshmi Y, Swetha Reddy L, Naga Neelima Devi K, Phani Kumar K, et al (2020) Evaluation of microneedling therapy in management of facial scars. J Craniofac Surg. https://doi.org/ 10.1097/scs.0000000000006145. [Epub ahead of print]

9. Pallua N, Kim BS (2020) Microfat and lipoconcentrate for the treatment of facial scars. Clin Plast Surg 47:139

10. Abdallah Hajj Hussein I, Dali Balta N, Jurjus RA et al (2012) Rat model of burn wound healing: effect of Botox. J Biol Regul Homeost Agents 26:389

11. Kuehlmann B, Stern-Buchbinder Z, Wan DC, Friedstat JS, Gurtner GC (2019) Beneath the surface: a review ofl aser remodeling of hypertrophic scars and burns. Adv Wound Care (New Rochelle) 8:168

12. Block L, Gosain A, King TW (2015) Emerging therapies for scar prevention. Adv Wound Care 4:607
13. https://www.grandviewresearch.com/press-release/global-scartreatment-market

14. Adler M, Mayo A, Zhou X, Franklin RA et al (2020) Principles of cell circuits for tissue repair and fibrosis. iScience 23:100841

15. Hewedy ES, Sabaa BEI, Mohamed WS, Hegab DS (2020) Combined intralesional triamcinolone acetonide and platelet rich plasma versus intralesional triamcinolone acetonide alone in treatment of keloids. J Dermatolog Treat 15:1

Publisher's Note Springer Nature remains neutral with regard to jurisdictional claims in published maps and institutional affiliations. 